\title{
Yoga in correctional settings: A randomized controlled study. Frontiers in Psychiatry. 2017 Oct 16; 8:204
}

\author{
Kalyan Maity* \\ Division of Yoga and Life Sciences, Swami Vivekananda Yoga Anusandhana Samsthana (S-VYASA), Bengaluru, Karnataka, India \\ Neuroscience Research Lab, Department of Neurology, PGIMER, Chandigarh, India
}

\author{
*Corresponding Author: \\ Kalyan Maity \\ Division of Yoga and Life Sciences, Swami Vivekananda Yoga Anusandhana Samsthana (S-VYASA), Bengaluru, \\ Karnataka, India \\ Contact no: +91-9481338079 \\ E-mail: maitykalyan769@gmail.com
}

\section{Introduction}

Yoga is a holistic approach and it is quite difficult to understand the specific effects of Yoga for its many aspects such as Asana, Pranayama, Meditation, etc. Inspite of these challenges, scientific studies on yoga have been growing optimally. Several studies have shown the positive outcome of Yoga on various psychological and physiological parameters such as stress, anxiety, depression, memory, aggressiveness, etc. $(1,2)$.

The prison population is vulnerable to different psychiatric morbidity and often represent antisocial behavior $(3,4)$. A randomized control trial has shown a significant improvement in perceived stress, positive effect, and psychological distress in prisoners after 10 weeks of yoga practice (5). A few meta-analyses have also suggested the positive effect of yoga and meditation on psychological well-being and behavioral functioning in the prison population.

The study has revealed that 10 weeks of Yoga is associated with increased positive effect, impulse control, psychological well-being, sleep quality, and decreased aggressiveness, stress, and negative effect.

\section{Study design}

Subjects who did not know the Swedish or English language and those whose stay was less than five months in prison were excluded from the study. Total 226 subjects (Male $n=201$, Female $n=25$ ) were recruited for the study from November 2013 to July 2015 and were randomly divided into Yoga group 134 (Male $\mathrm{n}=121$, Female $\mathrm{n}=13$ ) and Control group 92 (Male $\mathrm{n}=80$, Female $\mathrm{n}=12$ ). Subjects of the Yoga group practiced 90 minutes Yoga session once a week for ten weeks that included postures, breathing exercises, deep relaxation, and meditation techniques. Control group subjects practiced physical activity for the same duration as the Yoga group.
All subjects filled up a few questionnaires in Swedish as well as in English language before and after the intervention to measure their different psychological states. To measure stress level, they used the Perceived Stress Scale 14 Items (PSS-14) that consist of 14 items with Cronbach's alpha 0.84-0.86. Prison Adjusted Measure of Aggression (PAMA) was used to measure aggressiveness and antisocial behavior, which consisted 11 items having 6-point scoring scale from 0 to 5 with internal consistency between 0.67 \& 0.97. Positive and Negative Affect Schedule - Expanded From 30 Items (PANAS-X30) was administrated to measure positive and negative affect. This questionnaire comprised 30 items with internal consistency $0.80 \& 0.88$ for positive deactivated affects and $0.77 \& 0.85$ for negative deactivated affects. Conners' Continuous Performance Test II (CPT II) computer-based test was performed to check various types of attention and impulsivity. 19 items Pittsburgh Sleep Quality Index (PSQI) with good internal consistency 0.83 was administrated to measure the quality of sleep during the past month. Psychological symptoms and psychological distress were measured by Brief Symptom Inventory (BSI), which comprised 53 items, divided into 9 dimensions based on primary symptoms.

As data was not normally distributed, non-parametric test such as Fisher's exact test was used for the categorical variables and to compare the dropout rate between the group, Wilcoxon signed-rank test was done for within group comparisons. Mann-Whitney U tests was performed to check the level of significance $(\mathrm{P}<0.05)$ in between group analysis and Bivariate Spearman rank order correlation was conducted to measure the strength of association between yoga participants' expectation of yoga and few selected variables. Only the variables that matched the expectation in baseline assessment were taken for correlational analysis. 


\section{Implications}

Among 226 recruited inmates 74 dropped out from the study. Overall dropout rates were significantly higher in Yoga group $(42.5 \%)$ as compared to control group (18.5\%), whereas larger proportion of inmates left the study in control group (58.8\%) as compared to yoga group (28.1\%) due to their own request. Total 152 subjects (Male $n=133$, Female $n=$ 19; Yoga group $n=77$ and Control group $n=75$ ) successfully completed their follow-up. The result showed significant improvements in perceived stress, sleep quality, emotional and psychological well-being, antisocial behavior, aggressiveness, self-harm in Yoga group after 10 weeks of yoga practice. The control group also showed significant improvement in perceived stress and aggressiveness. Between-group comparison showed significant improvement in commissions, detectability, positive deactivated affect, negative activated affect, hit reaction time, and antisocial behavior with effect size ranged from 0.29-0.72.

No correlations were identified between outcome on selected variables and yoga participants expectation of yoga. Weak correlation coefficients showed that participants self-report did not affect their expectations of yoga.

Even though a few variables showed significant result in the between-group analyses but within group analyses showed most of the variables to be significant in the yoga group. Hence, it can be concluded that Yoga has a positive effect on jail inmates as compared to other physical activities.

\section{Inference}

Impaired executive function is directly associated with antisocial behavior. Yoga can be helpful to improve impulse control, attention and also reduced antisocial behavior. Prevalence of female prisoners are less as compare to male so gender-wise correlation was not possible. This study provides evidence in correctional sitting that included sleep quality. There was a chance to increase type 1 error because of multiple tests in this study. Major limitation of this study was low sample size, dropout and missing data that leads to low statistical power. Non-parametric tests were conducted because data was not normally distributed. Hence, it reduced statistical power and efficiency. Uses of different tests may misled the study. Even though dropout rate $(32.7 \%)$ in this study is less as compared to other Yoga based studies in correctional sitting but it was much higher than other Yoga studies. Only long-term participants should be included in future study to reduce dropout rate. Significantly younger inmates were not completed follow up. Hence, it is possible that younger inmates have more externalizing behavior and should be confirmed in future studies. Low Intervention frequency (Once in a week) and short follow-up period (10 weeks) could be the reason for not significant outcome in a few variables. A long follow up period and increased intervention frequency should be administrated in future studies.

\section{Informed consent}

Nil.

\section{Source of funding}

Nil.

\section{Conflict of interest}

Nil.

Received Date: 17-05-21; Revised Date: 06-07-21

Accepted Date: 08-07-21

\section{References}

1. Rocha KK, Ribeiro AM, Rocha KC, Sousa MB, Albuquerque FS, Ribeiro S, Silva RH. Improvement in physiological and psychological parameters after 6 months of yoga practice. Consciousness and cognition. 2012 Jun 1;21(2):843-50.

2. Deshpande S, Nagendra HR, Raghuram N. A randomized control trial of the effect of yoga on verbal aggressiveness in normal healthy volunteers. International journal of yoga. 2008 Jul;1(2):76.

3. Fazel S, Seewald K. Severe mental illness in 33588 prisoners worldwide: systematic review and meta-regression analysis. The British Journal of Psychiatry. 2012 May;200(5):364-73.

4. Falk Ö, Sfendla A, Brändström S, Anckarsäter H, Nilsson T, Kerekes N. Personality and trait aggression profiles of male and female prison inmates. Psychiatry research. 2017 Apr 1;250: 302-9.

5. Bilderbeck AC, Farias M, Brazil IA, Jakobowitz S, Wikholm C. Participation in a 10-week course of yoga improves behavioural control and decreases psychological distress in a prison population. Journal of psychiatric research. 2013 Oct 1;47(10):1438-45. 